\title{
Amygdala-Dependent Molecular Mechanisms of the Tac2 Pathway in Fear Learning
}

\author{
Raül Andero*, I,2,3, , Sarah Daniel ${ }^{2,3}$, Ji-Dong Guo ${ }^{2,3}$, Robert C Bruner ${ }^{2,3}$, Shivani Seth ${ }^{2,3}$, Paul J Marvar ${ }^{5}$, \\ Donald Rainnie $^{2,3}$ and Kerry J Ressler, ${ }^{*, 1,3}$
}

'Department of Psychiatry, McLean Hospital, Harvard Medical School, Belmont, MA, USA; 'Division of Behavioral Neuroscience and Psychiatric Disorders, Yerkes National Primate Research Center, Atlanta, GA, USA; ${ }^{3}$ Department of Psychiatry and Behavioral Sciences, Emory University School of Medicine, Atlanta, GA, USA; ${ }^{4}$ Institut de Neurociènces, Universitat Autònoma de Barcelona, Bellaterra, Spain; ${ }^{5}$ Department of Pharmacology and Physiology, George Washington University, Washington, DC, USA

\begin{abstract}
Recently we determined that activation of the tachykinin 2 (Tac2) pathway in the central amygdala (CeA) is necessary and sufficient for the modulation of fear memories. The Tac2 pathway includes the Tac2 gene, which encodes the neuropeptide neurokinin B and its corresponding receptor neurokinin 3 receptor (NK3R). In this study, using Tac2-cre and Tac2-GFP mice, we applied a combination of in vivo (optogenetics) and multiple in vitro techniques to further explore the mechanisms of action within the Tac2 pathway. In transgenic mice that express ChR2 solely in Tac2 neurons, in vivo optogenetic stimulation of CeA Tac2-expressing neurons during fear acquisition enhanced fear memory consolidation and drove action potential firing in vitro. In addition, Tac2-CeA neurons were shown to co-express striatal-enriched protein tyrosine phosphatase, which may have an important role in regulating Nk3R signaling during fear conditioning. These data extend our current understanding for the underlying mechanism(s) for the role of the Tac2 pathway in the regulation of fear memory, which may serve as a new therapeutic target in the treatment of fear-related disorders.

Neuropsychopharmacology (2016) 4I, 27I4-2722; doi: I 0. I038/npp.2016.77; published online 29 June 2016
\end{abstract}

\section{INTRODUCTION}

Altered fear learning in brain disorders such as post-traumatic stress disorder (PTSD), phobias, and obsessive-compulsive disorder can have serious consequences including tripling the rate of suicide (Kanwar et al, 2013). These disorders are also associated with huge economic costs for society (Olesen et al, 2012). The most common treatments for these conditions are psychotherapy, serotonin reuptake inhibitors, and anxiolytics; however, the effectiveness of such treatments is quite limited in many cases (Farb and Ratner, 2014).

Although there have been tremendous advances in the understanding of the neurocircuitry of fear learning (Herry and Johansen, 2014), there is still insufficient knowledge of the underlying mechanisms mediating fear processing, which is ultimately limiting the specificity and effectiveness of further therapeutic breakthroughs. The formation of fear memory following laboratory fear tasks has been studied according to the Pavlovian learning paradigm. This associative learning process consists of the pairing of a neutral

* Correspondence: Dr R Andero, Institut de Neurociènces, Universitat Autònoma de Barcelona, Bellaterra, Cerdanyola del Vallès, Barcelona 08193, Spain, Tel: +34 9358 I 3342, Fax: +34 93 58I 4I52,

E-mail: raul.andero@uab.cat or Dr KJ Ressler, Division of Depression \& Anxiety Disorders, McLean Hospital, Oaks Building 104b, Mailstop 212 , I I 5 Mill Street, Belmont, MA 02478- I064, USA, Tel: + I 61785542 I0, Fax: + I 617977 4213, E-mail: kressler@mclean.harvard.edu

Received 25 January 2016; revised 4 May 2016; accepted 8 May 20 16; accepted article preview online 26 May 2016 conditioned stimulus (CS) with an aversive unconditioned stimulus that elicits a conditioned fear response. The CS can be a cue (eg, a tone and auditory fear conditioning (FC)) or a context (eg, a room and contextual FC). The amygdala is one of the few brain regions that is considered a key nexus in networks of emotional memory, such as FC (Milad and Quirk, 2012).

There are several well-identified molecular mechanisms involved in fear memory formation. However, the most promising pathways for treating fear disorders are ones that can be pharmacologically targeted with safe and welltolerated drugs in humans. In line with this approach, we have recently uncovered the tachykinin 2 (Tac2) pathway as sufficient and necessary for the modulation of fear memories in mice (Andero et al, 2014). The Tac2 pathway includes neurokinin $\mathrm{B}(\mathrm{NkB})$, encoded by the Tac2 gene, and its specific receptor neurokinin 3 receptor (Nk3R) (Severini et al, 2002). We have shown an upregulation of Tac2 expression in the amygdala $30 \mathrm{~min}$ after auditory FC and an enhancement of fear memory consolidation with Tac2 lentiviral overexpression that is blocked by the selective Nk3R antagonist, Osanetant. Finally, Tac2 pharmacogenetic inactivation during $\mathrm{FC}$ reduces memory consolidation.

Tachykinins are abundant peptides in the central nervous system involved in neurotransmission and neuromodulation (Severini et al, 2002). Within the mouse amygdala, Tac2 is highly expressed within the central amygdala, especially in the medial division of the central nucleus (CeM) (Andero et al, 2014). The Nk3R is also highly expressed in the central 
amygdala in the mouse (Duarte et al, 2006). Interestingly, Nk3R antagonists, including Osanetant, are generally safe and well-tolerated drugs in humans (Malherbe et al, 2011), thus these findings could potentially be rapidly translated to the clinic. Hence, the Tac2 pathway may be of therapeutic interest in humans for brain disorders of fear regulation such as PTSD, phobia, panic, and obsessive-compulsive disorder. In this study, we provide additional evidence for the mechanism of the Tac2 pathway in the regulation of fear memories in mice.

\section{MATERIALS AND METHODS}

\section{Animals}

All experiments were performed on male adult wild-type strain C57BL/6J mice (WT), as well as the transgenic lines, B6.129-Tac2 < tm1.1(cre)Qima >/J (Tac2-cre) and B6.129 (Cg)-Tg(CAG-Beo/GFP)21Lbe/J (BGeo-GFP) from Jackson Labs. Tac2-GFP mice were obtained from crossing Tac2-cre mice and Bgeo-GFP mice. These mice were group-housed in a temperature-controlled vivarium, with ad libitum access to food and water. They were maintained on a $12 / 12 \mathrm{~h}$ light/ dark cycle, with all behavioral procedures being performed during the light cycle. All procedures used were approved by the Institutional Animal Care and Use Committee of Emory University and George Washington University, McLean Hospital, and in compliance with National Institutes of Health guidelines for the care and use of laboratory animals.

\section{Dual-Immunofluorescence Experiments}

Dual-immunofluorescence and immunofluorescence experiments were performed as previously described (Dabrowska and Rainnie, 2010) using 50- $\mu \mathrm{m}$-thick free-floating serial coronal brain sections (from Bregma -1.07 to $-1.79 \mathrm{~mm}$ ) from Tac2-GFP or WT mice. One to two mice were used for each combination of staining. The primary antibodies used were as follows: mouse monoclonal anti-PKC $\delta$ (1:500, 610398, BD Biosciences), mouse monoclonal anti-STEP (1:500, SC-23892, Santa Cruz), rabbit polyclonal antiNk3R (1:2000 in Tac2-GFP mouse and 1:1000 in WT mouse, (Griffond et al, 1997), and rabbit polyclonal anti-GFP antibody (1:1000, A-11122, Invitrogen, Carlsbad, CA, USA). Representative sections including the central amygdala were rinsed $3 \times$ for $10 \mathrm{~min}$ in PBS, permeabilized with $0.5 \%$ Triton-X 100 in PBS, and incubated for $48 \mathrm{~h}$ at $4{ }^{\circ} \mathrm{C}$ with the following primary antibody pairs in $0.5 \%$ Triton-X 100 in PBS with $1 \%$ BSA: PKC $\delta /$ GFP with Tac2-GFP tissue, STEP/ GFP with Tac2-GFP tissue, STEP/Nk3R with WT tissue, and Nk3R alone with Tac2-GFP tissue. Sections were rinsed $3 \times 10 \mathrm{~min}$ each in PBS and then incubated at room temperature for $2 \mathrm{~h}$ with Alexa-Fluor secondary antibodies specific for the primary antibody host: namely Alexa-Fluor 568 goat anti-mouse IgG and Alexa-Fluor 488 goat anti-rabbit IgG ( $1: 500$, Invitrogen). Procedures after incubation with secondary antibodies were followed as previously described (Dabrowska and Rainnie, 2010). Confocal spinning disk laser microscopy was used to analyze dual-immunoflurescence patterns (Dabrowska et al, 2013). The Tac2-GFP/NK3R lowmagnification pictures in the CeA were not optimal and not included here because of the very low signal in the red channel (Nk3R).

\section{Quantitative PCR (qPCR)}

These methods were followed as previously described (Andero et al, 2013). The primers were Tac3r Mm00445346_m1 and Ptpn5 Mm00479063_m1 from Applied Biosystems. These qPCR experiments were performed at Emory University and McLean Hospital. However, for $\mathrm{qPCR}$ in Figure $2 \mathrm{~g}$ and Supplementary Figure 3, the qPCR experiments were carried out at George Washington University and these methods are as follows: total RNA was isolated by TRIzol extraction (Invitrogen) and residual genomic DNA was removed by DNAse treatment (Turbo DNA-free, Invitrogen). Random hexamer-primed cDNA was generated using ImPromp-II reverse transcriptase (Promega, Madison WI). Reactions were assembled using a EpMotion 5070 liquid handling system (Eppendorf, Hauppauge, NY) that combined forward and reverse gene-specific primers $(0.3 \mu \mathrm{M}$ final concentration, Integrated DNA Technologies, with $7.5 \mu \mathrm{l}$ of SsoFast EvaGreen Supermix (Bio-Rad, Hercules, CA) in a $14 \mu \mathrm{l}$ reaction. qPCR analysis was performed using a CFX-384 Real-Time PCR Detection System. The Taqman primer for Tac3r was used for gene expression analysis.

\section{Stereotaxic Surgery}

Stereotaxic surgery was performed following similar procedures as previously described (Andero et al, 2013). Briefly, $1 \mu \mathrm{l}$ of pAAV-Ef1a-DIO-hChR2 (H134R)-EYFP-WPRE-pA was infused in the central amygdala of Tac2-cre mice at $1 \mathrm{nl} / \mathrm{s}$. Coordinates were as follows: anteroposterior, $-1.34 \mathrm{~mm}$; dorsoventral, $-4 \mathrm{~mm}$; mediolateral, $-2.4 \mathrm{~mm}$ relative to bregma. After each infusion, the injection cannula was allowed to remain for $2 \mathrm{~min}$. After the viral infection, and during the same surgery procedure, small holes were drilled into the skull and the optogenetic ferrule was implanted right above the CeM. Coordinates were as follows: anteroposterior, $-1.34 \mathrm{~mm}$; dorsoventral, $-4 \mathrm{~mm}$; mediolateral, $-2.4 \mathrm{~mm}$ relative to bregma. The optic fiber (Thorlabs) was chronically implanted and fixed to the skull using dental acrylic and jeweler's screws (Plastics One). Mice were allowed to recover for at least 6 weeks before testing. Following surgery, mice were handled weekly to habituate the mice to being restrained for removal of the dummy cap. After finishing the behavioral studies, mice were perfused with $4 \%$ paraformaldehyde. Brains were then equilibrated in $30 \%$ sucrose and sectioned on a cryostat. Visualization of the cannula placement was performed with the Zeiss Axioskop 2 plus microscope to verify its location.

\section{Cued Fear Conditioning, Fear Expression Tests, and Locomotor Activity}

Mice were given fear conditioning and tested for fear expression as previously described (Andero et al, 2014). Mice received two sessions of handling and habituation to the experimental box before conditioning. Each session was $5 \mathrm{~min}$ long. Conditioning consisted of five trials of a CS tone ( $30 \mathrm{~s}$, $6 \mathrm{kHz})$ co-terminating with a unconditioned stimulus footshock $500 \mathrm{~ms}, 6 \mathrm{~mA}$. The intertrial interval for conditioning 
was $90 \mathrm{~s}$. Fear expression was assessed $24 \mathrm{~h}$ after fear conditioning in the same chamber with changes to odor, light, and flooring to differentiate context and consisted of 15 CS tone trials with a $60 \mathrm{~s}$ intertrial interval. Tone presentation and freezing data were controlled, stored, and analyzed with FreezeView software (Coulbourn Instruments, Whitehall, PA). a Central Amygdala (CeA)
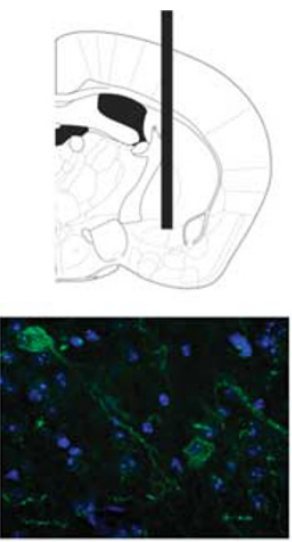

C

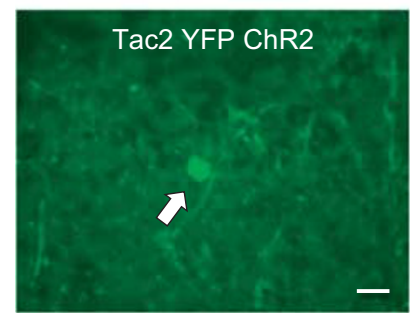

d

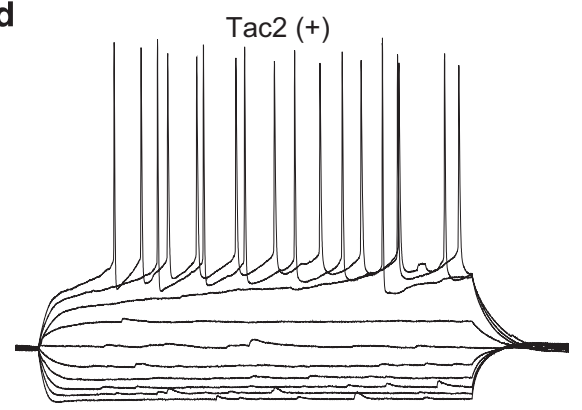

b

$10 \mathrm{~Hz}$
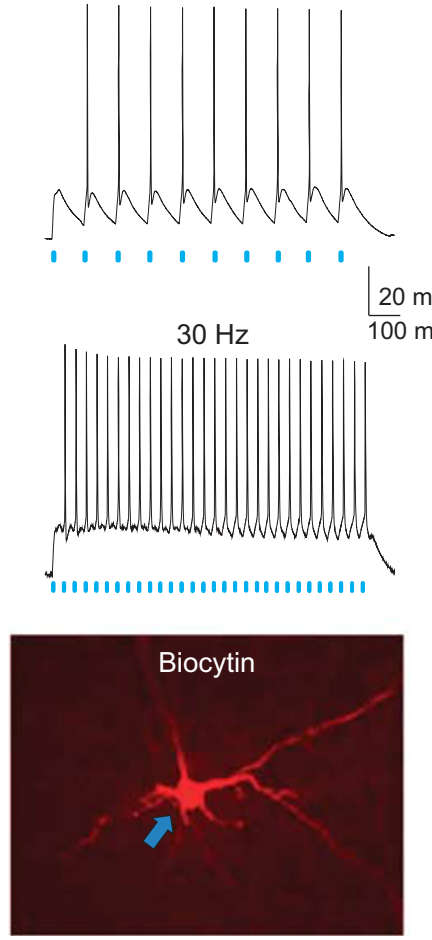

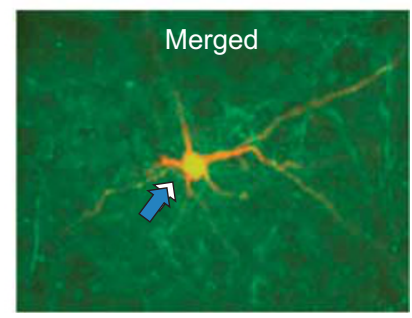

Tac2 (-)
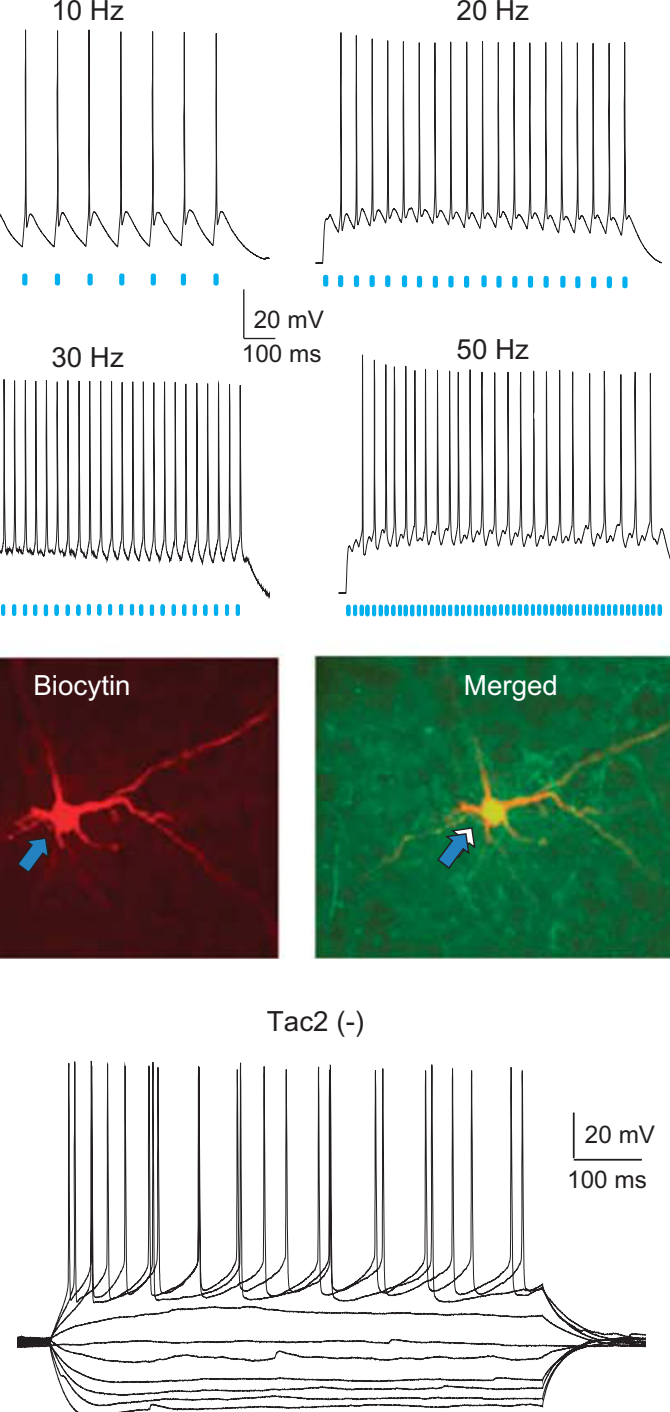
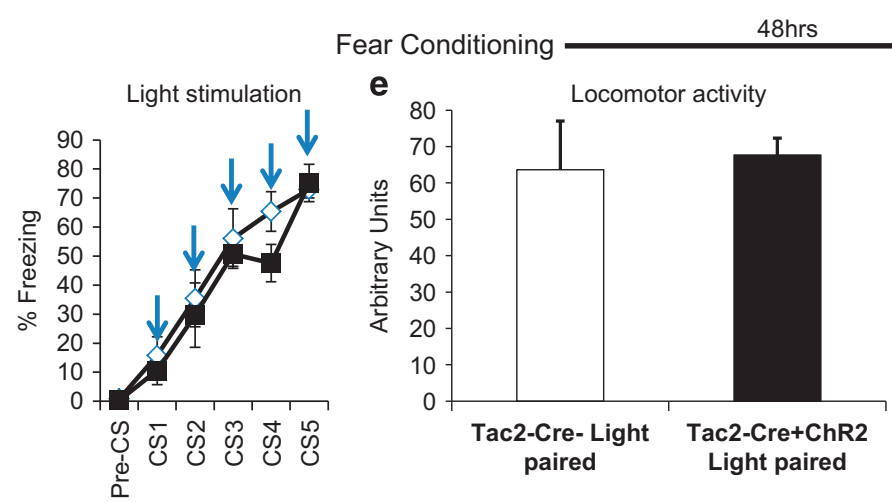
Fear Expression

f

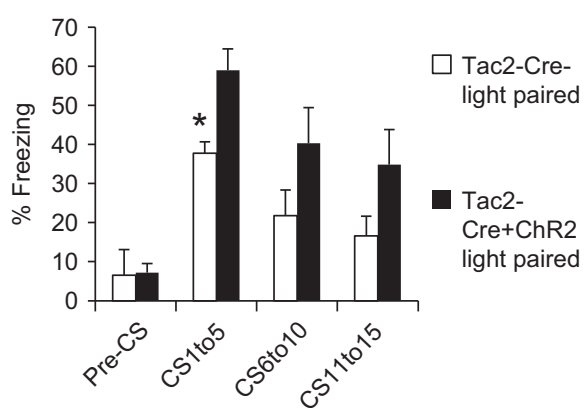

Figure I Optogenetic stimulation of central amygdala tachykinin 2 (Tac2)-expressing neurons drives action potential firing, in vitro, and enhances fear memory consolidation, in vivo. (a) The central amygdala (CeA) of Tac2-cre mice was unilaterally infected with DIO-ChR2 AAV. Top image represents the cannula used for delivering the AAV in the CeA. Bottom image shows the expression of the YFP reporter in Tac2 neurons within the central nucleus (CeM). (b) Light pulse trains at different frequencies. (c) Biocytin colocalizes with recorded CeM-Tac2-ChR2 neurons. Scale bar $20 \mu \mathrm{m}$. (d) Electrophysiological properties of Tac2-GFP-positive and Tac2-GFP-negative neurons in the CeM. (e and f) Optical stimulation of CeA-Tac2-ChR2 neurons during fear conditioning does not affect freezing levels or locomotor activity per se. $* p<0.05$. 
Locomotor activity during fear acquisition was measured by stopwatch by an experimenter blind to the groups. The arena was divided in nine equal quadrants. A transition from one quadrant to another, when the four paws of the animals were in the new quadrant, was considered as one arbitrary unit.

\section{Optogenetic Stimulation}

Optogenetic stimulation was performed using a 473-nm fiber optic-coupled laser (IkeCool). The laser-coupled fiber was connected to the optogenetic ferrule. Light stimulation parameters were $2 \mathrm{~s}$ stimulation at $20 \mathrm{~Hz}, 15 \mathrm{~ms}$ pulses. The estimated light intensity at the ends of the fibers were $15 \mathrm{~mW} / \mathrm{mm}^{2}$ with the 'Predicted irradiance values: model based on direct measurements in mammalian brain tissue' http://web.stanford.edu/group/dlab/cgi-bin/graph/chart.php. The stimulation was given during fear acquisition and stimulation co-terminated with the tone and shock (Jasnow et al, 2013).

\section{Slice Physiology}

Coronal brain slices ( $300 \mu \mathrm{m}$ thickness) containing the CeM of Tac2-cre mice expressing the flox-stopped ChR2 virus in the CeM were cut using a Leica VTS-1000 vibratome (Leica Microsystems, Bannockburn, IL, USA). Individual slices were incubated in a holding chamber containing artificial cerebrospinal fluid oxygenated with a mixture of $95 \%$ oxygen and $5 \%$ carbon dioxide at room temperature before recording (see (Jasnow et al, 2013) for details about slice preparation and patch clamping).

For whole cell patch clamp recording, slices were continuously perfused by gravity-fed oxygenated artificial cerebrospinal fluid heated to $32^{\circ} \mathrm{C}(1-2 \mathrm{ml} / \mathrm{min})$ in a Warner Series 20 submersion-type slice chamber $(0.5 \mathrm{ml}$ volume; Warner Instruments, Hamden, CT). Individual neurons in the slice were viewed using a Leica DM6000 FS microscope (Leica Microsystems) equipped with an IR-sensitive CCD camera (Orca ER, Hamamatsu, Tokyo, Japan). Tac2-GFP and Tac2-cre neurons expressing the ChR2 virus were visualized with $488 \mathrm{~nm}$ fluorescence illumination and targeted for whole-cell recording. Whole-cell recordings were made with a Multiclamp 700B amplifier (Molecular Devices Corporation, Sunnyvale, CA) using pClamp 10.4 software and an Axon Digidata 1550A-D interface (Molecular Devices Corporation). Current clamp signals were filtered at $5 \mathrm{KHz}$ and digitized at $10-20 \mathrm{KHz}$. Patch pipettes were fabricated from borosilicate glass (resistance 4-6 M $\Omega$ ) and filled with a standard potassium gluconate-based patch solution containing $0.3 \%$ biocytin.

A series of current clamp protocols was used to characterize Tac2 neurons. Briefly, a series of 10 hyperpolarizing and depolarizing square-wave current steps (750 ms) was injected to characterize the neurons' input resistance and firing properties, and a current ramp was injected to assess the threshold for action potential as previously described (Ehrlich et al, 2013). Membrane potential was held at $-60 \mathrm{mV}$ for all recordings. Membrane properties, including $I_{\mathrm{H}}$ ratio, $I_{\mathrm{K}(\mathrm{IR})}$ ratio, time constant (tau), input resistance $\left(R_{\mathrm{in}}\right)$, and spike characteristics, were analyzed using a custom MATLAB 2009a script (Mathwork, Natick, MA). The $I_{\mathrm{H}}$ ratio refers to a quantification of the hyperpolarizationactivated non-specific cation current, $I_{\mathrm{H}}$. When the membrane is hyperpolarized, the activation of $I_{\mathrm{H}}$ will depolarize the membrane in a voltage-dependent manner. The $I_{\mathrm{H}}$ ratio is the difference between the steady-state membrane potential at the end of the hyperpolarizing current step and the most negative membrane potential at the beginning of the step, divided by the most negative membrane potential. The $I_{\mathrm{K}(\mathrm{IR})}$ ratio refers to a quantification of the inward rectifying current, $I_{\mathrm{K}(\mathrm{IR})}$. The $I_{\mathrm{K}(\mathrm{IR})}$ ratio is calculated as the difference between the peak membrane potential at the beginning of the two smallest hyperpolarizing steps divided by the difference between the peak membrane potential at the beginning of the two largest hyperpolarizing steps, such that a cell with no observable inward rectification has a rectification ratio equal to 1 .

\section{Drugs Administration}

The Nk3R antagonist Osanetant (Axon Medchem) was dissolved in physiological saline and $0.1 \%$ Tween 20 which was also the vehicle. Vehicle or Osanetant were delivered intraperitoneally and tissue was harvested at the same time from both home cage and fear-conditioned mice. The dose of Osanetant was $5 \mathrm{mg} / \mathrm{kg}$ as previously described (Andero et al, 2014).

\section{Statistics}

Statistics were performed with IBM SPSS Statistics Version 19.0 (Armonk, NY) and GraphPad Prism 6.05 for Windows (GraphPad Software, La Jolla, CA). Detection of outliers was performed and removed from analyses when necessary. An outlier value was considered as higher or lower than two SD from the average. ANOVA or Student's $t$ test (two-tailed) were used where appropriate. The results are presented as means \pm SEM, and statistical significance was set at $p<0.05$.

\section{RESULTS}

\section{Electrophysiological Properties of Tac2-YFP-ChR2 Neurons and Tac2-GFP Neurons in the CeM}

Tac2-cre mice were injected with an AAV-floxed stop ChR2 virus-targeting CeM (Figure 1a). In all positive Tac2-YFPChR2 neurons tested, single-light pulses initially induced a membrane depolarization and subsequent action potential firing with higher light intensities (10-20 ms light pulse, $0.5-6 \mathrm{~mW} / \mathrm{mm}^{2}$ ). Significantly, high-fidelity spiking was induced in response to different frequencies of light pulse trains (Figure 1b), including the frequency $(20 \mathrm{~Hz})$ used in in vivo stimulations. Tac2 neurons also followed $30 \mathrm{~Hz}$ light pulse stimulation but exhibited significant failures when the frequency was increased to $50 \mathrm{~Hz}$. Figure 1c shows a Tac2ChR2-YFP-positive neuron that was fiilled with biocytin during recording for subsequent morphological reconstruction. See Supplementary Figure 1 for quantification of the firing response to the various frequencies of light pulse stimulation.

The physiological properties of Tac2-GFP-CeM neurons (obtained from crossing Tac2-cre mice and Bgeo-GFP mice) were characterized by recording their membrane properties 

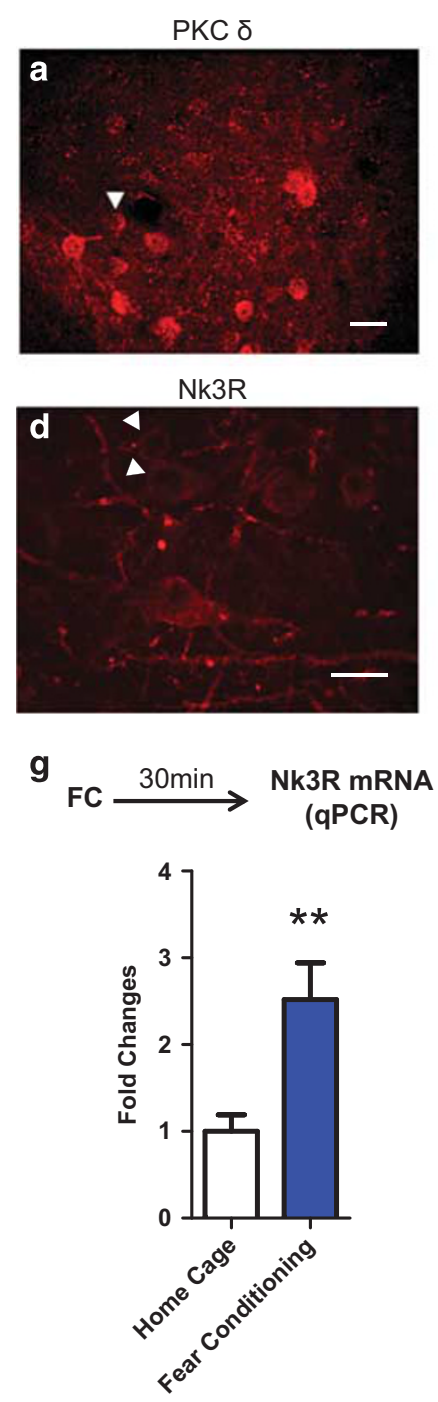
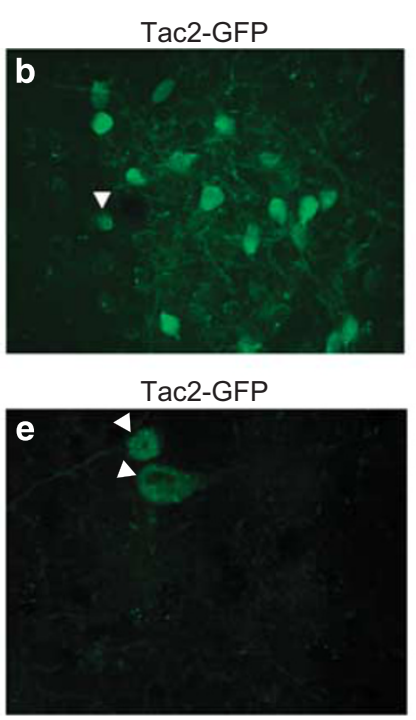

h
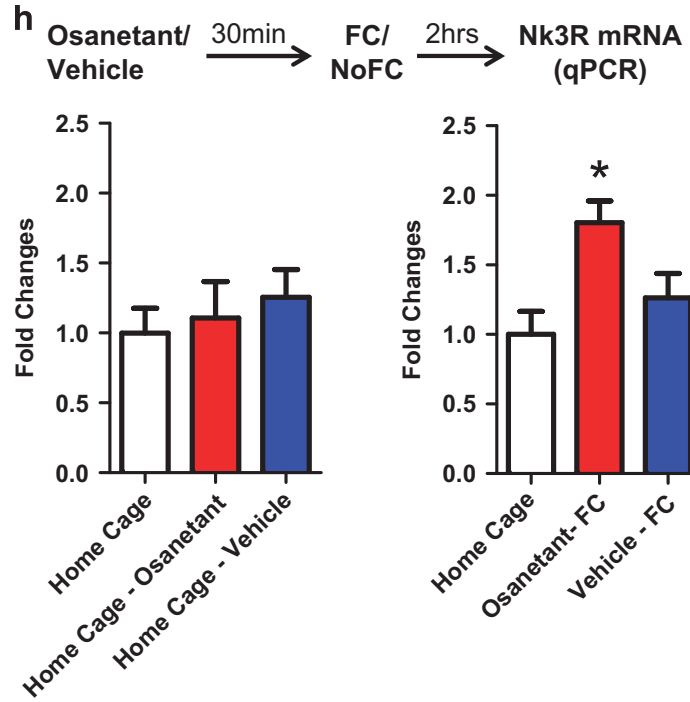
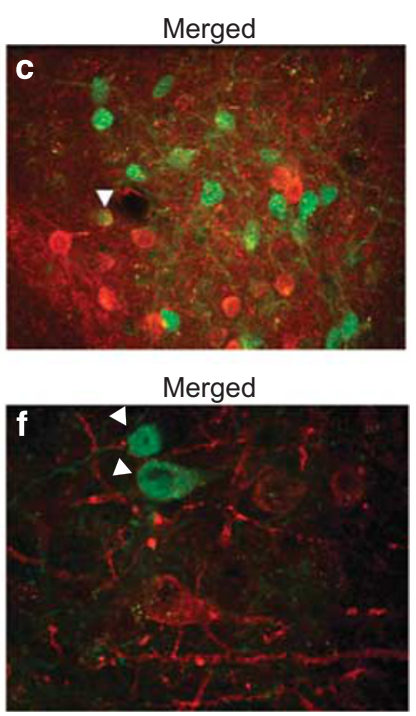

(qPCR)

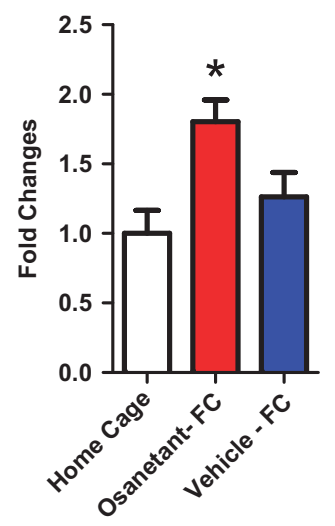

Figure 2 Immunohistochemistry studies of the tachykinin 2 (Tac2) neurons in the centromedial amygdala and association with the neurokinin 3 receptor in fear learning. (a) PKC $\delta$ neurons detected by immunohistochemistry. (b) Tac2-cre mice were crossed with a GFP fluorescent reporter mouse line, BGeo-GFP. (c) Some Tac2 neurons are colocalized with the PKC Tac2-cre-BGeo-GFP mice (Tac2-GFP). (f) Some Tac2 neurons are colocalized with Nk3R. (g) Nk3R mRNA levels are upregulated 30 min after auditory fear conditioning (FC) in C57 mice. (h) Wild-type mice received Osanetant (Nk3R antagonist) or vehicle. Two hours after FC, Nk3R mRNA levels were evaluated. ${ }^{*} p<0.05$; ** $p<0.01$. Scale bar for (a) $-(\mathrm{c})$ is $20 \mu \mathrm{m}, 40 \times$ images. Scale bar for (d) $-(\mathrm{f})$ is $20 \mu \mathrm{m}, 63 \times$ images.

(Figure 1d and Table 1). The location and GFP fluorescence of neurons were verified by including biocytin in patch solution and post hoc immunofluorescence procedures. Tac2-GFP-negative neurons that were adjacent to GFPpositive neurons were also recorded. As summarized in Table 1, Tac2 neurons exhibited basic membrane properties that were distinct from Tac2-negative neurons, including resting membrane potential $(t=5.161 \mathrm{df}=21$, and $p<0.001)$, $I_{\mathrm{H}}$ ratio $(t=3.705, \mathrm{df}=21$, and $p<0.01), I_{\mathrm{K}(\mathrm{IR})}$ ratio $(t=2.24, \mathrm{df}=21$, and $p<0.05), R_{\mathrm{in}}(t=2.730, \mathrm{df}=21$, and $p<0.05)$, action potential half width $(t=2.661, \mathrm{df}=21$, and $p<0.05)$, decay time $(t=3.893, \mathrm{df}=21$, and $p<0.001)$, and fast AHP $(t=2.647, \mathrm{df}=21$, and $p<0.05)$. Taken together, these data suggest that Tac2 neurons may represent a population of physiologically distinct neurons in the CeM.

\section{Behavioral Effects of Photostimulating Tac2-YFP-ChR2 Neurons}

Tac2-cre mice were injected with an AAV-floxed stop ChR2 virus-targeting CeM (same Materials and Methods as Figure 1a). Figure 1e (left) shows no effect in freezing during optical stimulation of Tac2-YFP-ChR2 neurons. In addition, there were no changes in locomotor activity during this fear acquisition testing (Figure 1e right). However, photostimulation of Tac2-YFP-ChR2 neurons during fear acquisition resulted in enhanced fear memory consolidation as shown by the significantly higher freezing levels of Tac2-YFP-ChR2 animals compared with Tac2-YFP animals during the fear expression test in the absence of any optogenetic stimulation (Figure 1f, CS1-5, $t=-3.677, \mathrm{df}=7$, and $p<0.05$ ). 
Table I Electrophysiological Properties of the Tac2 Neurons in the Centromedial Amygdala

\begin{tabular}{|c|c|c|c|c|c|c|c|c|c|c|}
\hline & \multirow[t]{2}{*}{ RMP (mV) } & \multirow[t]{2}{*}{$I_{\mathrm{H}}$ ratio } & \multirow[t]{2}{*}{$I_{K(I R)}$ ratio } & \multirow[t]{2}{*}{ Tau (ms) } & \multirow[t]{2}{*}{$\boldsymbol{R}_{\text {in }}(\mathrm{M} \Omega)$} & \multicolumn{5}{|c|}{ Spike } \\
\hline & & & & & & $\begin{array}{l}\text { Half width } \\
\text { (ms) }\end{array}$ & $\begin{array}{l}\text { Rise time } \\
\quad(\mathrm{ms})\end{array}$ & $\begin{array}{l}\text { Decay time } \\
\quad(\mathrm{ms})\end{array}$ & $\begin{array}{c}\text { Threshold } \\
(\mathrm{mV})\end{array}$ & fAHP $(\mathrm{mV})$ \\
\hline Tac2 YFP+ $(n=16)$ & $-66.4 \pm 0.7$ *** & $0.017 \pm 0.0017^{* *}$ & $3.21 \pm 0.30 *$ & $21.2 \pm 5.9$ & $166 \pm 13^{*}$ & $1.49 \pm 0.03 *$ & $0.49 \pm 0.025$ & $1.74 \pm 0.05 * *$ & $-30.2 \pm 0.96$ & $5.5 \pm 0.4 *$ \\
\hline Tac2 YFP - $(n=7)$ & $-57.0 \pm 2.3$ & $0.033 \pm 0.005$ & $2.14 \pm 0.24$ & $20.4 \pm 4.8$ & $350 \pm 101$ & $1.31 \pm 0.07$ & $0.45 \pm 0.01$ & $1.36 \pm 0.10$ & $-31.0 \pm 2.3$ & $10.0 \pm 2.5$ \\
\hline
\end{tabular}

*p $<0.05$; ** $p<0.01$ respectively vs Tac2 YFP-.

Table 2 Immunohistochemistry Studies of Tac2, Nk3R, PKC delta, and STEP in the Centromedial Amygdala

\begin{tabular}{lc}
\hline & Number of positive neurons \\
\hline Tac2 and PKC delta colocalization & 78 \\
GFP (Tac2) & 54 \\
Red (PKC delta) & 5 \\
Green and Red & $6.4 \%$ \\
Tac2 cells that express PKC delta & \\
& \\
Tac2 and STEP colocalization & 40 \\
GFP (Tac2) & 41 \\
Red (STEP) & 22 \\
Green and Red & $55 \%$ \\
Tac2 cells that express STEP & \\
& \\
NK3R and STEP colocalization & 66 \\
GFP (STEP) & 35 \\
Red (NK3R) & 30 \\
Green and Red & $85.7 \%$ \\
NK3R cells that express STEP & \\
\hline
\end{tabular}

Immunohistochemistry Studies of the Tac2 Neurons in the Centromedial Amygdala and Association with the Neurokinin 3 Receptor in Fear Learning

Figure $2 \mathrm{a}-\mathrm{c}$ shows that Tac2-GFP neurons in general do not colocalize with the protein kinase $\mathrm{C}$ delta $(\mathrm{PKC} \delta$ ) peptide, which has a key role in fear function (Haubensak et al, 2010). Quantification analysis shows that only $6.4 \%$ of Tac2 neurons express $\mathrm{PKC} \delta$ (Table 2). In contrast, Figure $2 \mathrm{~d}-\mathrm{f}$ suggests that some Tac2-GFP neurons are also Nk3Rimmunopositive (specific receptor of $\mathrm{NkB}$, the protein product of the Tac2 gene). Supplementary Figure 2 also shows that Tac2-GFP neurons also colocalize NkB.

We have previously shown that the Nk3R antagonist Osanetant given systemically or intra-CeM before and after FC impairs fear memory consolidation (Andero et al, 2014). Here we show that Nk3R mRNA levels were upregulated in the amygdala $30 \mathrm{~min}$ after FC in WT mice (Figure 2g, $t=4.138, \mathrm{df}=6$, and $p<0.01)$. In separate experiments, Osanetant or vehicle were given systemically $30 \mathrm{~min}$ prior to FC. Nk3R mRNA levels were upregulated $2 \mathrm{~h}$ after FC in the group of mice that received Osanetant (Figure $2 \mathrm{~h}$ right, ANOVA $f=4.869 \mathrm{df}=20$, and $p<0.05$; Bonferroni $p<0.05$ home cage $v s$ Osanetant FC). The group of mice that had received vehicle before FC presented similar Nk3R mRNA levels to the home cage group. Control experiments show that neither vehicle nor Osanetant changed Nk3R mRNA levels (Figure $2 \mathrm{~h}$ left). In summary, shortly after FC Nk3R, mRNA levels are upregulated (30 min after FC, Figure $2 \mathrm{~g}$ ) returning later to baseline levels ( $2 \mathrm{~h}$ after $\mathrm{FC}$ vehicle group, Figure $2 \mathrm{~h}$ ). However, this normal expression of the Nk3R mRNA levels after FC is altered when Osanetant is dosed ( $2 \mathrm{~h}$ after FC Osanetant group, Figure $2 \mathrm{~h}$ ). In addition, we evaluated Nk3R and Tac2 mRNA levels in other areas important in fear regulation (Supplementary Figure 3). As shown in panel a of Supplementary Figure 3, there is a trend for an upregulation of Tac2 mRNA levels 30 min after fear conditioning in the paraventricular nucleus of the hypothalamus but no changes in levels of NkR3 mRNA. Nk3R and Tac2 mRNA levels in the bed nucleus of the stria terminalis did not change $30 \mathrm{~min}$ after fear conditioning. These results provide further evidence for the molecular mechanisms and neural circuits involved in the Tac2/NkR3 pathway and may further explain the effects of Osanetant on fear memory consolidation (Andero et al, 2014).

\section{Immunohistochemistry Studies of the Tac2 Neurons in the Centromedial Amygdala and Association with Striatal-Enriched Protein Tyrosine Phosphatase in Fear Learning}

Several studies have associated the Tac2 pathway with the modulation of memory formation (Andero et al, 2014; Chao et al, 2014; de Souza Silva et al, 2013); however, downstream signaling pathways that may interact with Tac2 are not well-known. Striatal-enriched protein tyrosine phosphatase (STEP) is a brain-specific tyrosine phosphatase that has a key role in processes of stress and memory (Dabrowska et al, 2013; Karasawa and Lombroso, 2014), and is expressed at high levels in the CeA. Notably, STEP has been shown to oppose the development of synaptic plasticity and fear memory consolidation within the amygdala (Paul et al, 2007). We examined whether STEP is colocalized in Tac2 neurons. Figure $3 \mathrm{a}-\mathrm{c}$ shows that STEP is indeed colocalized with some Tac2-GFP neurons in the CeM. Quantification analysis shows that $55 \%$ of Tac 2 neurons express STEP and $85.7 \%$ of Nk3R neurons express STEP, Table 2. See Figure $3 \mathrm{~d}-\mathrm{f}$ for images of STEP-expressing neurons showing colocalization with the Nk3R (Supplementary Figure 4).

STEP mRNA levels were shown to be downregulated 30 min after FC in WT mice (Figure $3 \mathrm{~g}, t=2.941, \mathrm{df}=5$, and $p<0.05)$. In separate experiments, mice received vehicle or Osanetant $30 \mathrm{~min}$ before FC to further examine STEP/NK3R interactions. STEP mRNA levels, measured $2 \mathrm{~h}$ after FC, 

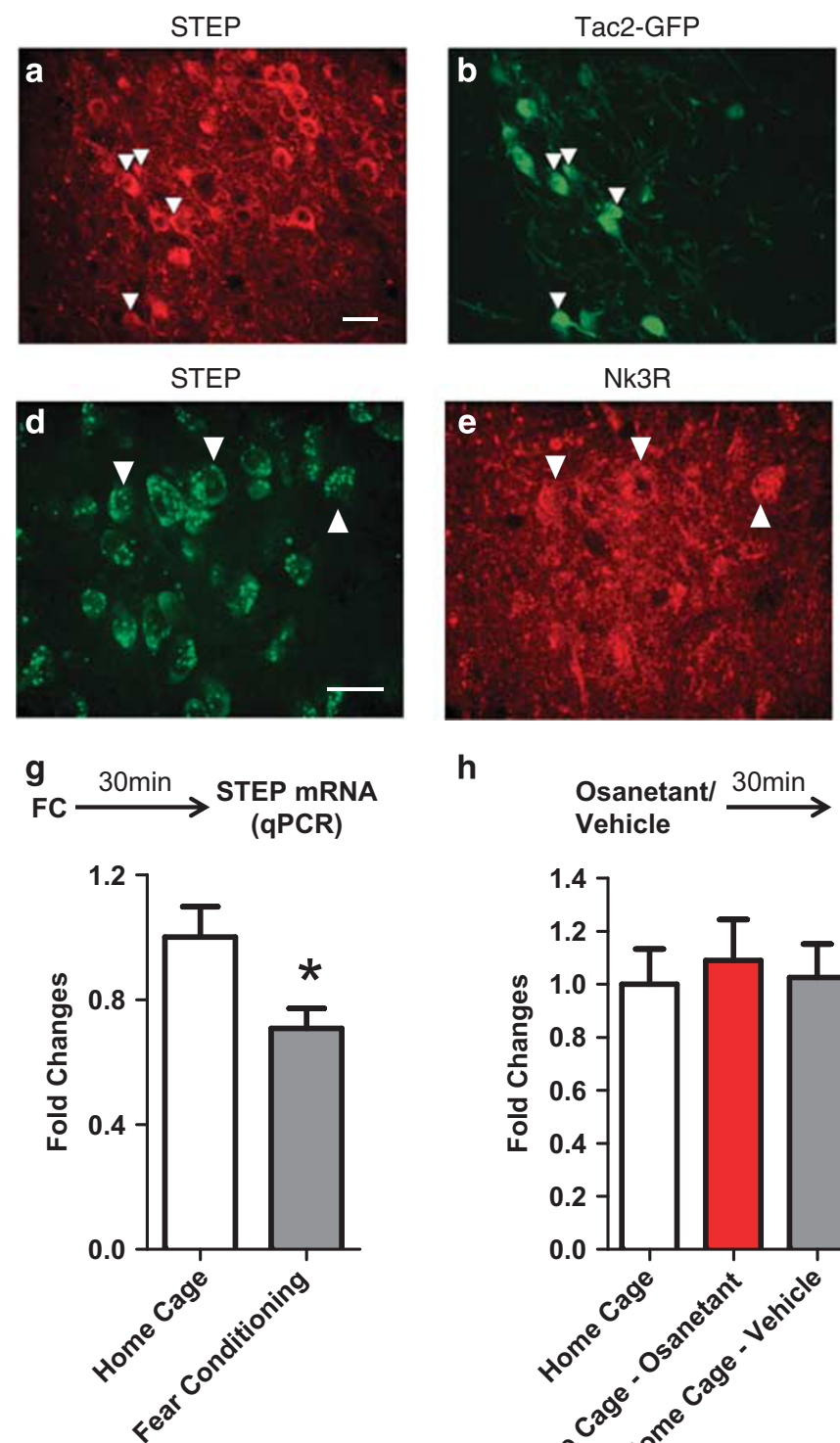

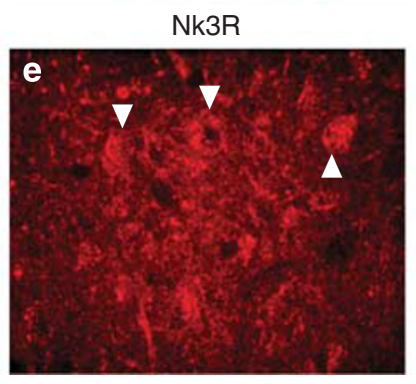

h
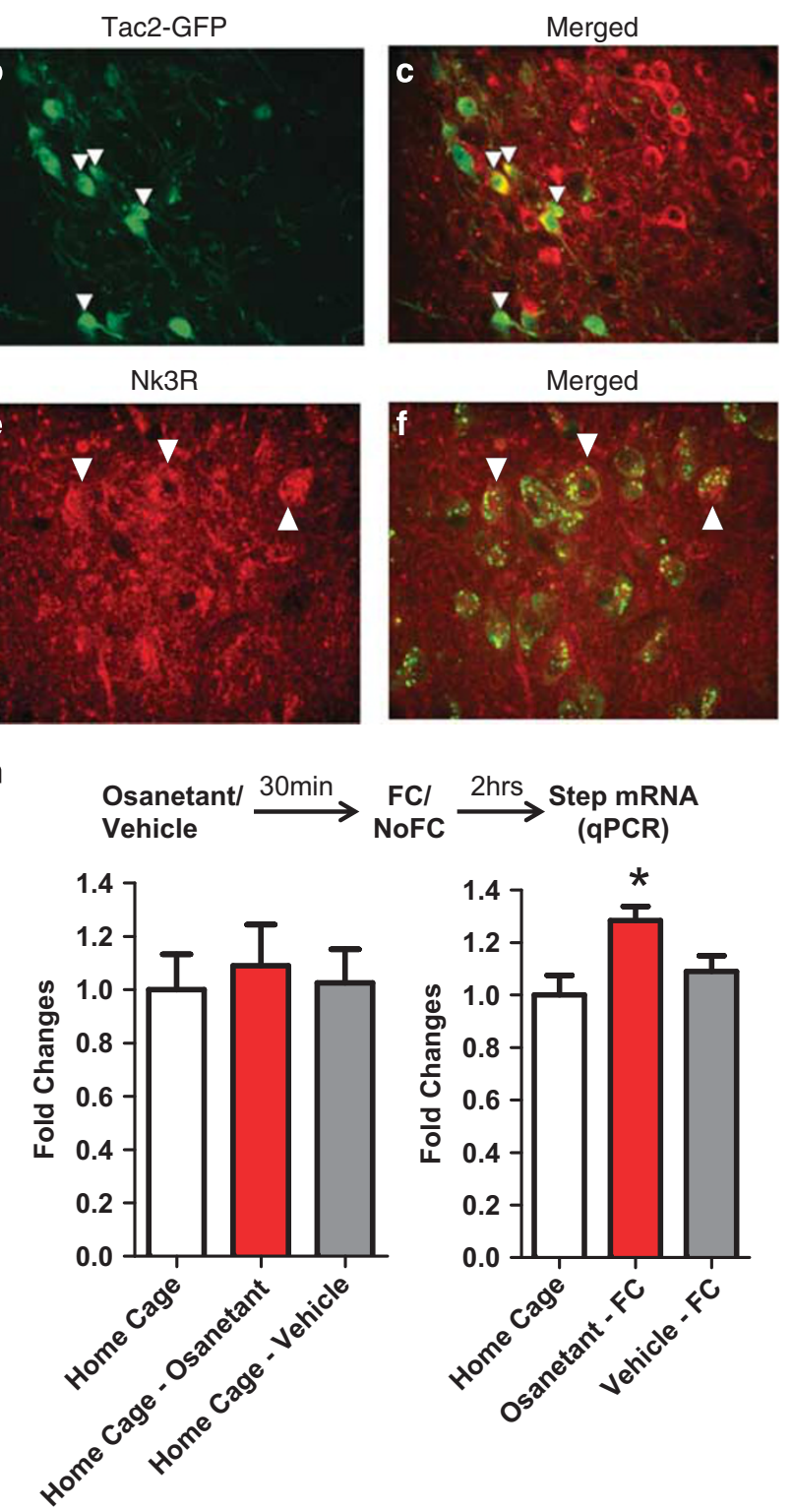

Figure 3 Immunohistochemistry studies of the tachykinin 2 (Tac2) neurons in the central medial amygdala and association with striatal-enriched protein tyrosine phosphatase in fear learning. (a) Striatal-enriched protein tyrosine phosphatase (STEP) neurons detected by immunohistochemistry. (b) Tac2-cre mice were crossed with a GFP fluorescent reporter mouse line and BGeo-GFP. (c) Some Tac2 neurons are colocalized with the STEP protein. (d) STEP neurons detected by immunohistochemistry. (e) Detection of Nk3R neurons with immunohistochemistry. (f) Some STEP neurons are colocalized with Nk3R. (g) STEP mRNA levels are downregulated 30 min after auditory fear conditioning (FC) in wild-type (WT) mice. (h) WT mice received Osanetant (Nk3R antagonist) or vehicle. Two hours after FC STEP mRNA levels were evaluated. $* p<0.05$. Scale bar for (a) - (b) is $20 \mu \mathrm{m}, 40 \times$ images. Scale bar for (d) -(f) is $20 \mu \mathrm{m}, 63 \times$ images.

were upregulated in the group of mice that had received Osanetant (Figure $3 \mathrm{~h}$, ANOVA $f=4.605, \mathrm{df}=23$, and $p<0.05$; Bonferroni $p<0.05$ home cage $v s$ Osanetant FC). Thus, in the vehicle group, STEP mRNA levels were rapidly downregulated (30 min after FC, Figure $3 \mathrm{~g}$ ) returning later to baseline levels ( $2 \mathrm{~h}$ after FC vehicle group; Figure $3 \mathrm{~h}$, right). Interestingly, Osanetant seemed to alter the normal expression of STEP mRNA levels after FC because STEP mRNA levels were upregulated $2 \mathrm{~h}$ after FC (Osanetant FC group; Figure $3 \mathrm{~h}$ right). Control experiments showed that neither vehicle nor Osanetant changed STEP mRNA levels in the absence of FC (Figure 3h, left).

\section{DISCUSSION}

These results build upon our previous findings and provide new evidence for Tac2 signaling as a novel molecular pathway in fear regulation within the mouse amygdala (Andero et al, 2014). Our data suggests that the Tac2 neurons within the CeM have a different electrophysiological profile from their neighbor neurons that are non-Tac2-expressing cells. For example, the lower resting membrane potential and input resistance of Tac2 neurons suggests that these neurons will require more excitatory input to be activated than neighboring neurons. Importantly, in vitro photostimulation of amygdala-Tac2-YFP-ChR2 neurons validates the 
stimulation protocol used in vivo, as these neurons are able to follow a $20 \mathrm{KHz}$ stimulation.

In vivo optogenetic stimulation of amygdala-Tac2-YFPChR2 results in no changes in freezing during fear acquisition nor locomotor activity. However, changes in freezing during the fear expression test after photostimulation of amygdala-Tac2-YFP-ChR2 during fear acquisition suggests that it may enhance fear memory consolidation. This opens many new questions for future experiments, such as examining the CeA-Tac2 projections to other areas related to fear memories including the paraventricular nucleus of the hypothalamus and bed nucleus of the stria terminalis, as well as investigating interactions with other neuropeptide systems involved in fear learning such as angiotensin II, oxytocin, or opiods (Andero, 2015; Bealer and Flynn, 2003; Hurt et al, 2015; Marvar et al, 2014).

PKC $\delta$-labeled neurons have been suggested to be part of a microcircuit in which these centro-lateral amygdala neurons, called $\mathrm{CeL}_{\text {off }}$ units, inhibit neuronal output to the CeM during the CS, thereby inhibiting fear expression (Haubensak et al, 2010). In our previous studies, we found that Tac2 mRNA and $P K C \delta$ mRNA levels are not colocalized within the CeM (Andero et al, 2014). Here, we extend these findings showing that Tac2-GFP neurons do not colocalize with the $\mathrm{PKC} \delta$ peptide in the CeM. Together, these data may suggest that Tac2-CeM may be an independent circuit from $\mathrm{PKC} \delta$.

Here we show that Tac2-GFP and Nk3R are colocalized in the CeM. This could suggest that NkB release acts in an autocrine and paracrine manner in synaptic regulation. The FC experiments suggest that Nk3R levels are modulated in the amygdala both by $\mathrm{FC}$ and the Nk3R antagonist Osanetant. Of note, our previous studies have shown that Osanetant given systemically or intra-CeM results in impaired auditory FC (Andero et al, 2014). These data suggest that upon binding of $\mathrm{NkB}$ to $\mathrm{Nk} 3 \mathrm{R}$, there could be an internalization of the receptor that causes synthesis of new Nk3R resulting in a short-term increase in Nk3R mRNA. Osanetant occupation of the Nk3R may inhibit NkB binding, resulting in blockade of memory consolidation and a robust and long-lasting increase in the synthesis of Nk3R mRNA. It is also possible that Nk3R in other areas outside the CeA could be affected by a systemic injection of Osanetant.

We also found that STEP is colocalized with Tac2-GFP and Nk3R within the CeM. In addition, STEP mRNA levels are decreased at $30 \mathrm{~min}$ after FC. This is in line with previous findings that show an association of STEP with a fear memory paradigm (Paul et al, 2007). In another experiment, Osanetant and vehicle were given systemically 30 min before FC, and STEP mRNA levels were evaluated $2 \mathrm{~h}$ later. The group of mice that had received Osanetant presented enhanced levels of STEP when compared with the home cage group. However, no changes in STEP mRNA levels were found when comparing the home cage group and the vehicle group. An explanation of these findings could be that STEP is acutely downregulated after FC $(30 \mathrm{~min})$ returning to baseline levels ( $2 \mathrm{~h}$ after FC). The increase in STEP mRNA expression $2 \mathrm{~h}$ after FC with Osanetant suggests that the normalization of STEP mRNA levels is initiated by a separate signal not affected by Osanetant. Blocking the Nk3R with Osanetant potentially prevents the acute reduction in STEP mRNA without preventing the slow increase in STEP mRNA that would normalize expression, resulting in an increase in
STEP mRNA $2 \mathrm{~h}$ after FC. Alternatively, Osanetant may more directly alter the normal expression pattern of STEP after FC through a different mechanism. This alteration could be associated with the impairment in fear memory consolidation that occurs when Osanetant is dosed in mice (Andero et al, 2014). These results suggest that the Nk3R modulates STEP levels during fear memory consolidation. Interestingly, it has been proposed that STEP downregulates ERK1/2 activity during fear conditioning (Paul et al, 2007). ERK1/2 is a key for gene transcription in memory formation.

In brief, we report new evidence that furthers our understanding for the mechanisms underlying Tac2dependent cellular pathways in fear learning. These findings may have broad implications in the understanding of fear memory functions in the healthy brain and new treatments in psychiatric disorders that present pathological fear learning, eg, PTSD, phobias, or panic.

\section{FUNDING AND DISCLOSURE}

RA and KJR declare intellectual property of the patent PCT/ US2015/037629. The remaining authors declare no conflict of interest.

\section{ACKNOWLEDGMENTS}

We would like to thank Dr Philippe Ciofi, INSERM (France), for the donation of the $\mathrm{NkB}$ and Nk3R antibodies. RA and KJR were supported by R21MH101492-01 and RA by a NARSAD Young Investigator Grant (22434) and the Ramón y Cajal programme (RYC-2014-15784). PJM was supported by NIH R00 HL107675.

\section{REFERENCES}

Andero R (2015). Nociceptin and the nociceptin receptor in learning and memory. Prog Neuropsychopharmacol Biol Psychiatry 62: 45-50.

Andero R, Brothers SP, Jovanovic T, Chen YT, Salah-Uddin H, Cameron $\mathrm{M}$ et al (2013). Amygdala-dependent fear is regulated by Oprl1 in mice and humans with PTSD. Sci Transl Med 5: 188 ra173.

Andero R, Dias BG, Ressler KJ (2014). A role for Tac2, NkB, and $\mathrm{Nk} 3$ receptor in normal and dysregulated fear memory consolidation. Neuron 83: 444-454.

Bealer SL, Flynn FW (2003). Central neurokinin 3 receptors increase systemic oxytocin release: interaction with norepinephrine. Exp Neurol 184: 1027-1033.

Chao OY, Nikolaus S, Huston JP, de Souza Silva MA (2014). The neurokinin-3 receptor agonist senktide facilitates the integration of memories for object, place and temporal order into episodic memory. Neurobiol Learn Mem 114: 178-185.

Dabrowska J, Hazra R, Guo JD, Li C, Dewitt S, Xu J et al (2013). Striatal-enriched protein tyrosine phosphatase-STEPs toward understanding chronic stress-induced activation of corticotrophin releasing factor neurons in the rat bed nucleus of the stria terminalis. Biol psychiatry 74: 817-826.

Dabrowska J, Rainnie DG (2010). Expression and distribution of $\mathrm{Kv} 4$ potassium channel subunits and potassium channel interacting proteins in subpopulations of interneurons in the basolateral amygdala. Neuroscience 171: 721-733.

de Souza Silva MA, Lenz B, Rotter A, Biermann T, Peters O, Ramirez A et al (2013). Neurokinin3 receptor as a target to 
predict and improve learning and memory in the aged organism. Proc Natl Acad Sci USA 110: 15097-15102.

Duarte CR, Schutz B, Zimmer A (2006). Incongruent pattern of neurokinin B expression in rat and mouse brains. Cell Tissue Res 323: 43-51.

Ehrlich DE, Ryan SJ, Hazra R, Guo JD, Rainnie DG (2013). Postnatal maturation of GABAergic transmission in the rat basolateral amygdala. J Neurophysiol 110: 926-941.

Farb DH, Ratner MH (2014). Targeting the modulation of neural circuitry for the treatment of anxiety disorders. Pharmacol Rev 66: 1002-1032.

Griffond B, Ciofi P, Bayer L, Jacquemard C, Fellmann D (1997). Immunocytochemical detection of the neurokinin $\mathrm{B}$ receptor (NK3) on melanin-concentrating hormone $(\mathrm{MCH})$ neurons in rat brain. J Chem Neuroanat 12: 183-189.

Haubensak W, Kunwar PS, Cai H, Ciocchi S, Wall NR, Ponnusamy R et al (2010). Genetic dissection of an amygdala microcircuit that gates conditioned fear. Nature 468: 270-276.

Herry C, Johansen JP (2014). Encoding of fear learning and memory in distributed neuronal circuits. Nat Neurosci 17: 1644-1654.

Hurt RC, Garrett JC, Keifer OP Jr, Linares A, Couling L, Speth RC et al (2015). Angiotensin type 1a receptors on corticotropinreleasing factor neurons contribute to the expression of conditioned fear. Genes Brain Behav 14: 526-533.

Jasnow AM, Ehrlich DE, Choi DC, Dabrowska J, Bowers ME, McCullough KM et al (2013). Thyl-expressing neurons in the basolateral amygdala may mediate fear inhibition. J Neurosci 33: 10396-10404.

Kanwar A, Malik S, Prokop LJ, Sim LA, Feldstein D, Wang Z et al (2013). The association between anxiety disorders and suicidal behaviors: a systematic review and meta-analysis. Depress Anxiety 30: 917-929.

Karasawa T, Lombroso PJ (2014). Disruption of striatal-enriched protein tyrosine phosphatase (STEP) function in neuropsychiatric disorders. Neurosci Res 89: 1-9.

Malherbe P, Ballard TM, Ratni H (2011). Tachykinin neurokinin 3 receptor antagonists: a patent review (2005-2010). Expert Opin Ther Pat 21: 637-655.

Marvar PJ, Goodman J, Fuchs S, Choi DC, Banerjee S, Ressler KJ (2014). Angiotensin type 1 receptor inhibition enhances the extinction of fear memory. Biol Psychiatry 75: 864-872.

Milad MR, Quirk GJ (2012). Fear extinction as a model for translational neuroscience: ten years of progress. Annu Rev Psychol 63: 129-151.

Olesen J, Gustavsson A, Svensson M, Wittchen HU, Jonsson B group, C.s., European Brain, C. (2012). The economic cost of brain disorders in Europe. Eur J Neurol 19: 155-162.

Paul S, Olausson P, Venkitaramani DV, Ruchkina I, Moran TD, Tronson N et al (2007). The striatal-enriched protein tyrosine phosphatase gates long-term potentiation and fear memory in the lateral amygdala. Biol Psychiatry 61: 1049-1061.

Severini C, Improta G, Falconieri-Erspamer G, Salvadori S, Erspamer V (2002). The tachykinin peptide family. Pharmacol Rev 54: 285-322. 